\title{
Masa escrotal como resultado de la extensión de un pseudoquiste pancreático
}

\author{
Ballestero Diego R, Aguilera Tubet C, Gutiérrez Baños JL, Martín García B, Portillo Martín JA, \\ Zubillaga Guerrero S.
}

Servicio de Urología. Hospital Universitario Marqués de Valdecilla. Santander.

Actas Urol Esp. 2008;32(2):261-264

\section{RESUMEN}

MASA ESCROTAL COMO RESULTADO DE LA EXTENSION DE UN PSEUDOQUISTE PANCREATICO

Las causas de orquitis son diversas y entre ellas se encuentran los procesos inflamatorios. Nosotros queremos comunicar el caso de un varón joven con clínica y exploración de orquitis, originada esta por la extensión de un pseudoquiste pancreático, no sospechado en un principio y descubierto por técnicas de imagen.

Palabras clave: Orquitis. Pseudoquiste pancreático. TC abdominal.

\section{ABSTRACT \\ SCROTAL MASS AS RESULT OF THE EXTENSION OF A PSEUDOCYST PANCREATIC}

There are several causes for orchitis and among them there are inflammatory process. We want to communicate the case of a young man with a clinical history and physical examination compatible with orchitis produced by the extension of a pancreatic pseudocyst that was not suspected in the beginning and discovered by imaging procedures.

Keywords: Orchitis. Pancreatic pseudocyst. CT abdominal.

$\mathrm{E}^{1}$ páncreas es una glándula de secreción endocrina y exocrina en la parte superior del retroperitoneo. Las lesiones pancreáticas pueden producir de forma secundaria colecciones a nivel local capaces de extenderse a tejidos y órganos adyacentes. La afectación de los espacios perirrenales puede alcanzar la superficie anterolateral del músculo psoas, proporcionando un plano de drenaje inferior que puede alcanzar la pelvis e incluso las extremidades inferiores. Sin embargo la aparición de este tipo de lesiones no es habitual.

Un cuadro de orquitis con funiculitis importante en un paciente con historia previa de pancreatitis y elevación de la amilasa debe hacernos sospechar la presencia de un pseudoquiste pancreático. El TAC en un método muy efectivo de diagnóstico.

Presentamos el caso de un paciente, con historia previa de pseudoquiste pancreático controlado, que presenta clínica de orquitis e intensa funiculitis.

\section{CASO CLÍNICO}

Paciente de 38 años que acude al Servicio de Urgencias por dolor en teste izquierdo con irradiación a fosa iliaca izquierda. Como antecedentes personales destacar únicamente una pancreatitis aguda flemonosa y exudativa complicada estadio D hace 5 años. Durante su seguimiento por parte del Servicio de Medicina Interna se objetiva trombosis de vena esplénica y colección peripancreática en región pararrenal anterior izquierda de unos $5 \mathrm{~cm}$, sin cambios en los últimos controles anuales.

El paciente refiere dolor sordo en teste izquierdo con irradiación a fosa iliaca izquierda, sin sindrome miccional asociado. A la exploración, aumento de la bolsa escrotal izquierda, dolorosa a la palpación con aparente aumento del tamaño testicular izquierdo, ausencia de diferenciación epidídimo-testicular y funiculitis asociada. La analítica sanguínea, urinaria y la radiografia de abdomen revelan resultados normales. El paciente refiere 
una semana de tratamiento con ciprofloxacino vía oral sin mejoría. Se añade gentamicina intramuscular y un antiinflamatorio en el contexto de una supuesta orquitis.

El paciente regresa al Servicio de Urgencias 3 días después por mala respuesta al tratamiento anterior. Refiere el mismo dolor de la primera visita junto a picos febriles de hasta $38,2{ }^{\circ} \mathrm{C}$. A la exploración, se observa una zona empastada y dolorosa en fosa iliaca izquierda sin signos de peritonismo. La exploración testicular revela los mismos signos que en la anterior exploración, con menor dolor a la palpación testicular pero con un cordón espermático más engrosado y doloroso. La nueva analítica sanguínea continúa con valores normales salvo una amilasa de 371 Unid/dL.

Ante la mala evolución de la orquitis con el tratamiento pautado y la elevación de la amilasa sanguinea, se realiza una ecografia abdominal la cual es informada como engrosamiento del páncreas y esplenomegalia con abundante circulación colateral. Los testículos y epidídimos son normales, destacando una colección rodeando al testículo izquierdo que se extiende por el canal inguinal y rodea al cordón espermático al cual inflama. Ante la sospecha de que todo el cuadro haya sido provocado por una reagudización de su pancreatitis y crecimiento del pseudoquiste se completa el estudio con la realización de un TAC abdominopélvico que es informado como agenesia de lóbulo hepático izquierdo, gran esplenomegalia y abundante circulación colateral. Páncreas edematoso con una colección peripancreática que se extiende por el espacio pararrenal anterior izquierdo, continuando por delante del psoas iliaco, saliendo por el canal inguinal izquierdo $\mathrm{y}$ engrosando el cordón espermático provocando una funiculitis secundaria a pancreatitis. (Figs. $1,2,3,4$ y 5)

Ante el hallazgo de una orquitis y funiculitis irritativa en el contexto clínico de un pseudoquiste pancreatico, el paciente es derivado al Servicio de Digestivo para completar su estudio y su tratamiento posterior.

\section{DISCUSIÓN}

Una de las complicaciones de la pancreatitis es la formación de un pseudoquiste pancreático.

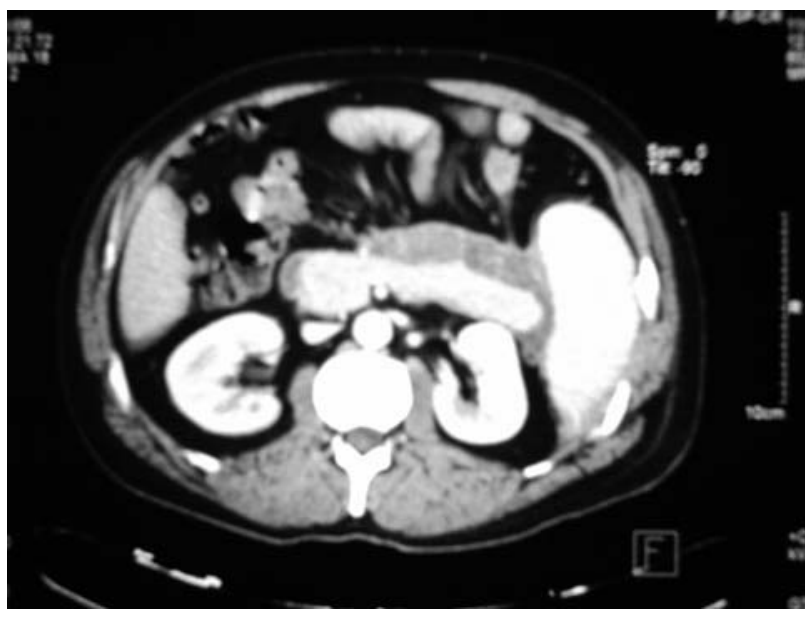

FIGURA 1. Colección peripancreática.

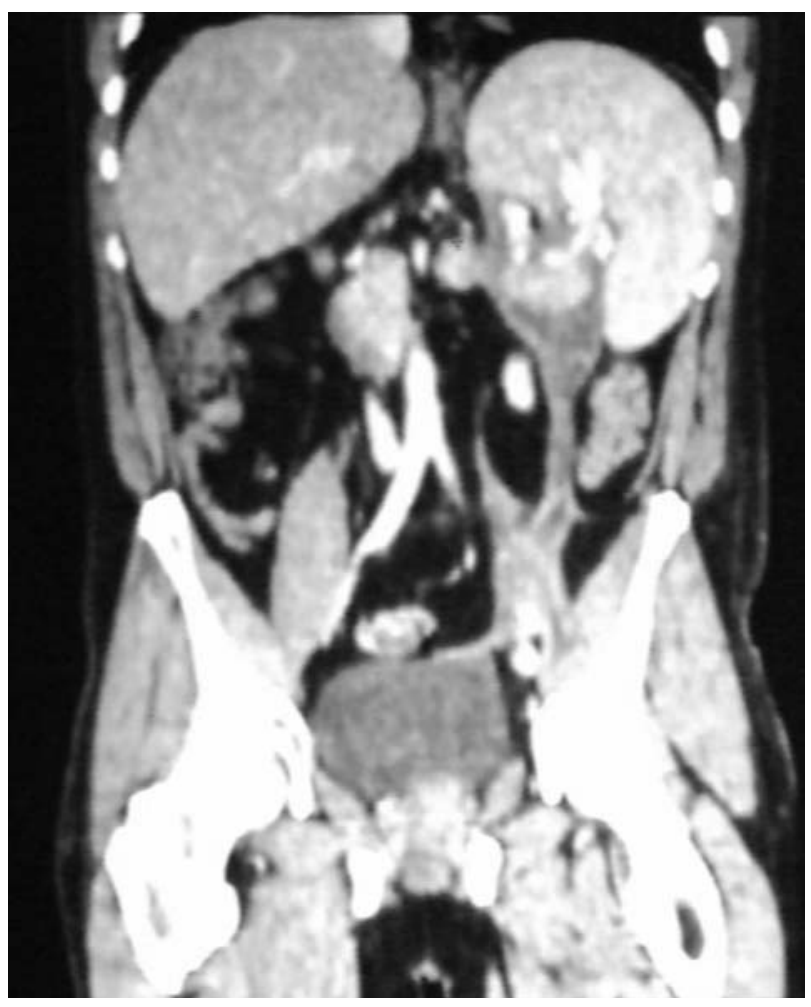

FIGURA 2. Colección sobre músculos psoas.

Suele ser el resultado de una pancreatitis alcohólica ${ }^{1}$. Se producen como consecuencia de la formación de una brecha en el conducto pancreático con la consecuente salida de líquido pancreático con sus enzimas alrededor del tejido. Estas colecciones son capaces de extenderse a espacios y órganos vecinos. De hecho, la extensión de procesos retroperitoneales hacia el escroto se debe a la disposición de los mismos; Pueden hacerlo a través del compartimento formado por 


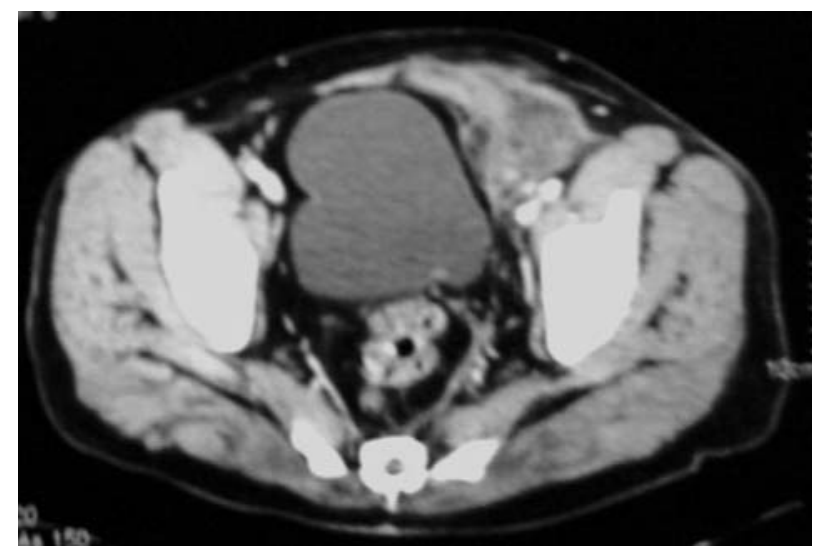

FIGURA 3. Colección en región inguinal izquierda.



FIGURA 4. Colección peritesticular izquierda.

la fascia de Gerota (anterior) y la de Zuckerland (posterior). Asímismo entre la fascia de Gerota y el peritoneo parietal posterior existe un espacio virtual que puede comunicar el retroperitoneo con el escroto, al igual que si persiste el conducto peritoneo-vaginal abierto ${ }^{2,3}$.

En otras ocasiones se han descrito casos en que el pseudoquiste ha ascendido a través del hiato esofágico o aórtico al mediastino y al cuello ${ }^{4-6}$.

El pseudoquiste pancreático se trata de una complicación rara. Howard y Jordan et al, después de analizar varios estudios, concluyen que la incidencia de esta patología constituye el 0,007 por ciento de las admisiones hospitalarias ${ }^{7}$.

A diferencia de la colecciones peripancreáticas, los pseudoquistes están rodeados de una pared formada por fibrosis y tejido de granulación. Estas cavidades pueden infectarse y dar lugar a lo que se conoce como absceso pancreático. El líquido contenido en el pseudoquiste pre-

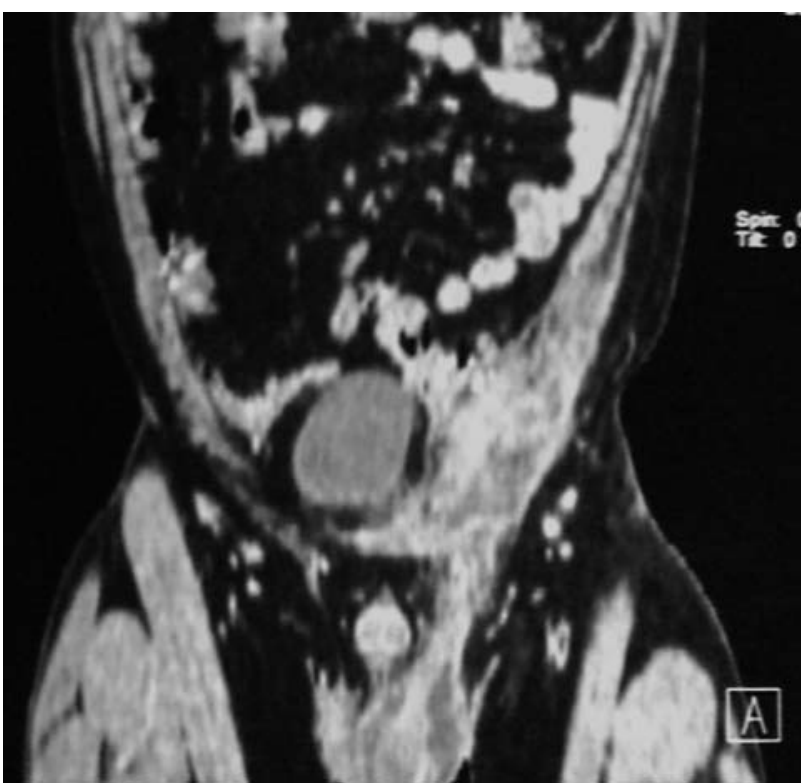

FIGURA 5. Extensión de colección inguinal izquierda.

senta altas concentraciones de enzimas pancreáticos y su crecimiento suele ser el resultado de una reagudización subclínica.

La mayor parte de los casos aportados a la literatura se tratan de masas inguinales dolorosas que simulan una hernia incarcerada. Al realizar su exploración quirúrgica, los autores se encontraron con áreas de necrosis grasa y una cantidad elevada de enzimas pancreáticas en el líquido drenado ${ }^{6,8,9}$. El diagnóstico se llevó a cabo mediante la administración de contraste a través de la fistula cutánea, lo cual demostró su comunicación con el páncreas. En nuestro caso, el diagnóstico se realizó a través de un TAC. También se ha descrito la extensión escrotal de la pancreatitis que debutó con signos de una torsión testicular ${ }^{3}$.

Un tratamiento aceptable del pseudoquiste pancreático consiste en el drenaje percutáneo de la colección, teniendo una tasa de curaciones del $90 \%{ }^{1}$. Esta opción es atractiva debido a su minima invasión y a la facilidad para realizarla. Algunas contraindicaciones para el drenaje percutáneo son: presencia de necrosis pancreática, masa sólida pancreática, falta de ruta segura de acceso y hemorragia intraquística activa.

Otras opciones cuando el drenaje percutáneo no es exitoso o esta contraindicado, son el manejo endoscópico a través de un drenaje transpapilar mediante un stent o bien creando una comu- 
nicación con el quiste transgástrica, la resección quirúrgica o bien la realización de alguna de las técnicas de marsupialización tanto por vía abierta como laparoscópica ${ }^{10}$.

En nuestro caso, el síntoma guía fue el dolor escrotal con inflamación del testículo que simulaba una orquitis. Después de iniciado un tratamiento antibiótico y tras la ausencia de mejoria clínica evidente, se sometió al paciente a la realización de una ecografía y TAC que confirman el crecimiento del pseudoquiste ya conocido en el paciente hasta el escroto. El tratamiento posterior fue conservador.

Creemos interesante la comunicación de este caso para recordar que la aparición de una masa escrotal o inguinal en un paciente con historia de alcoholismo y un cuadro más o menos reciente de pancreatitis, debe hacernos sospechar la presencia de un pseudoquiste pancreático.

\section{REFERENCIAS}

1. Yeo CJ. Pancreatic pseudocyst, ascites, and fistulas. Curr Opin Gen Surg. 1994;173-178.

2. Lye DJ, Stark RH, Cullen GM, Wepfer JF. Ruptured pancreatic pseudocyst: extension into the thigh. AJR Am J Roentgenol. 1987;149(5):937-938.
3. Lin YL, Lin MT, Huang GT, Chang YL, Chang H, Wang SM, et al. Acute pancreatitis masquerading as testicular torsion. Am J Emerg Med. 1996;14(7):654-655.

4. Sybers HD, Shelp WD, Morrissey JF. Pseudocyst of the pancreas with fistulous extension into the neck. N Engl J Med. 1968;278(19):1058-1059.

5. Bergmann F, Jacobs E, Malaisse W, Thys JP. Fistulation pleurate d'un pseudocyste pancréatique. Acta Chir Belg 1965; Suppl 3:128-135.

6. Steedman RA, Doering R, Carter R. Surgical aspects of pancreatic abscess. Surg Gynecol Obstet. 1967;125(4): 757-762.

7. Howard JH, Jordan GL: Surgical Diseasses of the Pancreas, Philadelphia, Lippincott, 1960.

8. Erzurum VZ, Obermeyer R, Chung D. Pancreatic pseudocyst masquerading as an incarcerated inguinal hernia. South Med J. 2000;93(2):221-222.

9. Salvo AF, Nematolahi H. Distant dissection of a pancreatic pseudocyst into the right groin. Am J Surg. 1973;126(3): 430-432.

10. Yin WY. The role of surgery in pancreatic pseudocyst. Hepatogastroenterology 2005;52(64):1266-1273.

Correspondencia autor: Dr. R. Ballestero Diego Servicio de Urología

Hospital Universitario Marqués de Valdecilla Avenida de Valdecilla, s/n - 39009 Santander Tel.: 942202520

E-mail autor: badiro@yahoo.es Información artículo: Nota clínica

Trabajo recibido: mayo 2006

Trabajo aceptado: octubre 2007 